\title{
Hepatotoxicity of combined therapy with rifampicin and daily prothionamide for leprosy
}

\author{
JI BAOHONG,${ }^{*}$ CHEN JIAKUN, ${ }^{*}$ WANG \\ CHENMIN† \& XIA GUANG $\dagger$ \\ *Zeng Yi Hospital, Shanghai, and †Hai-an Leprosy Hospital, \\ Jiangsu Province, People's Republic of China
}

Accepted for publication 31 October 1983

\begin{abstract}
Summary Liver injury was observed in $56 \%$ of 39 leprosy patients treated with combinations of dapsone, prothionamide (PTH), and isopiperazinylrifamycin SV in Hai-an, and in $22 \%$ of 50 patients treated with a combination of dapsone, rifampicin (RMP), PTH and clofazimine in Shanghai. Fatalities occurred among both groups of patients after 3 or 4 months of combined chemotherapy. The drug responsible for liver injury was probably $\mathrm{PTH}$, although RMP administered simultaneously may have been a contributing factor. It appears necessary to examine liver function monthly during the first 6 months of treatment by a combined drug regimen that includes PTH.
\end{abstract}

\section{Introduction}

In its fifth report, ${ }^{1}$ the WHO Expert Committee on Leprosy emphasized the need to prevent the development of drug resistance, and recommended that all active cases of multibacillary leprosy be treated with at least 2 effective anti-leprosy drugs. Subsequently, because the prevalence of dapsone resistance, both primary and secondary, had been steadily increasing in many leprosy-endemic countries, the WHO Study Group on Chemotherapy of Leprosy for Control Programmes further recommended, ${ }^{2}$ that at least 2 additional drugs be combined with dapsone for treatment of multibacillary leprosy, and that one of these drugs be rifampicin. Following these recommendations, we embarked on a clinical trial of combined chemotherapy in Hai-an County, Jiangsu Province, and in Shanghai. A high incidence of liver injury was observed after 1-3 months of treatment, accompanied by several deaths.

\section{Hai-an}

Thirty-nine patients were treated, 29 males and 10 females, whose ages ranged 
from 26 to 88 years (median age 47 years), and who included 18 patients with LL leprosy, 18 with $\mathrm{BL}$, and 3 with $\mathrm{BB}$ leprosy. The duration of disease ranged from 1 to 35 years (median duration 16 years); only 2 were 'new' patients, the remaining 37 having been treated with dapsone as monotherapy for from 3 to 227 months (median duration 57 months). The general condition of the patients was fair; body weights ranged from 45 to $70 \mathrm{~kg}$ (median weight $62 \mathrm{~kg}$ ); liver and renal function were normal before the initiation of combined therapy.

The patients were divided into 3 groups. Those of Group A, 22 in number, were treated with dapsone $100 \mathrm{mg}$ and prothionamide (PTH) $300 \mathrm{mg}$, each administered daily, together with R-76-1 (isopiperazinylrifamycin SV3) $300 \mathrm{mg}$, on each of the first 2 days of treatment and once monthly thereafter. The 13 patients of Group B were treated with dapsone $100 \mathrm{mg}$ and PTH $300 \mathrm{mg}$ daily, together with R-76-1 $300 \mathrm{mg}$ on each of the first 14 days and $600 \mathrm{mg}$ once monthly thereafter. The 4 patients of Group C were treated with PTH, either $300 \mathrm{mg}$ (3 patients) or $500 \mathrm{mg}$ (1 patient) daily as monotherapy for the first 90 days of treatment, in order to measure the initial rate of killing of Mycobacterium leprae by PTH; thereafter, their treatment was supplemented with dapsone $100 \mathrm{mg}$ daily together with R-76-1 $300 \mathrm{mg}$ daily on 2 consecutive days every month. Administration of all of the drugs was supervised. The duration of combined therapy varied somewhat among individual patients; the median duration was 97 days among Group A patients, 99 days among the patients of Group B, and 105 days among those of Group C. The median total dosages of dapsone, PTH and R-76-1 per patient were, respectively, $9700 \mathrm{mg}, 28,800 \mathrm{mg}$, and $1500 \mathrm{mg}$ for Group A patients, $9900 \mathrm{mg}, 29,100 \mathrm{mg}$, and $5400 \mathrm{mg}$ for Group B patients, and for the patients of Group C, dapsone $1500 \mathrm{mg}$, PTH 31,200 mg (3 patients) and $52,000 \mathrm{mg}$ (1 patient), and R-76-1 $600 \mathrm{mg}$.

Gastrointestinal side-effects, among them anorexia, nausea and vomiting, occurred in $17(44 \%)$ patients. In fact, almost all patients complained of loss of appetite in some degree. The nausea and vomiting usually occurred about 30 minutes after ingesting the drugs, and endured $1-5 \mathrm{~h}$. Because these side-effects also occurred in 2 of the 4 patients of Group C, they appear to have been caused mainly by PTH. The gastrointestinal side-effects subsided during continued therapy in only 1 patient, and became progressively worse in 11 patients, all of whom were found to have evidence of liver damage. Thus, it appears that the gradual worsening of the gastrointestinal side-effects resulted from liver damage as well as from local irritation of the gastrointestinal tract.

Fifteen $(39 \%$ ) patients became jaundiced between 24 and 120 days (median 96 days) after initiation of combined therapy. In 5 patients, jaundice appeared only 5-20 days after therapy had been suspended.

Because urobilirubin or urobilinogen was found in the urine of $28(72 \%)$ patients about 90 days after initiation of therapy, liver and renal function were examined in all patients. Renal function was entirely normal. However, serum glutamic-pyruvic transaminase (SGPT) levels were found to be elevated in 19 
patients: 7 in the range $41-100$ units; 6 in the range 101-200 units; 3 in the range 201-300 units, and 3 in the range 301-384 units. Fourteen patients - all except for 5 of the 7 whose elevation of the SGPT was in the lowest range-developed jaundice, either before SGPT levels were measured or subsequently. In 3 additional patients, elevations of icterus index or serum bilirubin or a positive flocculation reaction were detected, accompanied in all by urobilirubin or urobilinogen in the urine. Thus, a total of $22(56 \%)$ patients showed laboratory evidence of liver damage- 12 from Group A, 7 from Group B and 3 from Group C. The frequency of liver damage among the 3 treatment groups was, for Group A $55 \%$, for Group B $54 \%$, and for Group C $75 \%$; the differences among the treatment groups are not significant $(P>0.05)$. Such a high frequency of liver damage was unexpected.

Combined therapy was immediately suspended in all cases. In all but 2 patients, the liver function tests returned to normal within 60 days. One patient, No. 977, died during convalescence from liver damage, the cause of death apparently acute haemorrhagic necrotic enteritis. A second patient, No. 166, showed progressive jaundice despite suspension of therapy, became comatose and died. A brief history of this patient's illness is given.

This 63-year-old male with LL leprosy began combined therapy (Group A) on 23 March 1982. On 4 May 1982, 42 days after beginning treatment, he complained of anorexia and gastric discomfort. On 27 June 1982, jaundice was recognized, and the patient complained of profound anorexia accompanied by nausea and vomiting. Laboratory studies at this time revealed: icterus index 120, serum bilirubin $13.6 \mathrm{mg} \%$, SGPT 306 units, HBsAg negative. His jaundice progressed, and his liver rapidly became smaller, as shown by physical and ultrasound examinations. On 1 July 1982, petaechial haemorrhages and haematemesis occurred, and the patient became comatose. Two days later, 102 days after initiation of combined therapy and 6 days after the onset of jaundice, the patient died in hepatic insufficiency. An autopsy was not performed.

\section{Shanghai}

Fifty patients, 31 males and 19 females ranging in age from 22 to 69 years (median age 44 years), were treated in Shanghai. Forty-one were classified as LL, 8 as BL, and 1 as BB. Duration of disease ranged from 5 to 48 years (median duration 26 years). All had been treated with dapsone as monotherapy for periods ranging from 48 to 324 months (median duration 288 months). Their general condition was fair, and body weights ranged from 52 to $76 \mathrm{~kg}$ (median weight $59 \mathrm{~kg}$ ); liver function tests were normal before beginning combined chemotherapy.

The single combined regimen consisted of dapsone $100 \mathrm{mg}$ and PTH $300 \mathrm{mg}$, each self-administered daily, together with rifampicin (RMP) $900 \mathrm{mg}$, PTH 500 $\mathrm{mg}$, and clofazimine (CLO) $300 \mathrm{mg}$, each administered monthly under super- 
vision. The duration of combined therapy was 30 days for 24 patients, and 31-50 days for the remaining 26 patients. The median total dosages of RMP and CLO per patient were, respectively, 900 and $300 \mathrm{mg}$ for the first group, and $1800 \mathrm{mg}$ and $600 \mathrm{mg}$ for the second.

Gastrointestinal side-effects were encountered in $32(64 \%)$ of the patientsprimarily anorexia, nausea and vomiting. These side-effects became more striking immediately after the monthly supplemental doses had been administered. One patient became jaundiced about 30 days after initiation of combined therapy, whereupon liver function was examined in all patients between 30 and 50 days after beginning therapy. Liver damage was detected in $11(22 \%)$ patients, 8 of whom showed elevations of the SGPT -5 in the range $41-100$ units, and 3 in the range 101-200 units. Three additional patients demonstrated elevation of the icterus index and serum bilirubin. Combined therapy was suspended in all patients, and liver function tests were repeated. Among the 11 patients with evidence of liver damage, 9 had recovered by 30 days after the suspension of therapy, but the remaining 2 patients revealed elevations of the SGPT - to 80 and 168 units - 60 days after therapy had been suspended.

The frequency and severity of liver damage were less among the Shanghai than among the Hai-an patients, perhaps because of the shorter duration of combined therapy administered to the former group. However, acute hepatic insufficiency occurred in another patient after treatment for 4 months with PTH $300 \mathrm{mg}$ daily and RMP $900 \mathrm{mg}$ monthly. A brief history of this patient's illness is the following.

This 62-year-old male, with leprosy classified as LL and with dapsone resistance demonstrated by mouse foot-pad inoculation, began combined chemotherapy on 19 March 1982. On 23 July 1982, 126 days later, he complained of anorexia and nausea and was noted to be slightly jaundiced. Therapy was immediately suspended. Laboratory study revealed icterus index 72 , serum bilirubin $7.0 \mathrm{mg} \%$, and SGPT $>400$ units. In spite of treatment, jaundice progressed, and on 1 August 1982, the patient became comatose; at this time, his blood ammonia was measured at $200 \mu \mathrm{g} \%$. Three days later, 138 days after beginning combined therapy and 12 days after the onset of jaundice, the patient died in hepatic insufficiency. At autopsy, acute hepatic necrosis was discovered.

\section{Discussion}

The prevalence of secondary dapsone resistance among patients with multibacillary leprosy in the Shanghai area was recently shown to be greater than $8 / 100$ patients at risk, ${ }^{4}$ and primary resistance to dapsone has recently been detected both in Shanghai and in the Hai-an area (Ji Baohong et al., unpublished data). Thus, the need to adopt combined therapy for leprosy is urgent. However, there has been some uncertainty with respect to the choice of appropriate combined 
drug regimens, based on considerations of safety, efficiency and operational feasibility, as evidenced by the many multidrug regimens that have been recommended since 1976. The regimens selected for the present study comply with the basic requirement laid down by the WHO Study Group, ${ }^{2}$ i.e. they include at least 2 effective drugs in addition to dapsone, one of which is RMP. Because many Chinese patients do not accept CLO, PTH was also employed in these regimens.

The liver damage observed in the course of the present study had the following characteristics: (1) it appeared almost simultaneously among the patients under treatment, and did not occur among patients treated with dapsone as monotherapy during the same period; (2) most patients recovered within 30-60 days after the therapy was suspended; and (3) of 15 patients from the Hai-an area and 9 from Shanghai examined for evidence of $\mathrm{HBsAg}$ and $\mathrm{HBcAg}$, only one of the first group was positive for HBsAg. Thus, viral hepatitis, at least that caused by the hepatitis B virus, may be excluded as an important cause of the liver damage encountered in the present study. On the other hand, toxic hepatitis, caused by the combined therapy employed in this study, appears to be the only explanation for the liver damage observed.

There is very little information in the literature with respect to the hepatotoxicity of combined regimens containing both PTH and RMP. In the treatment of tuberculosis, PTH had been largely abandoned at the time that RMP became available. However, one study has been reported. It has been reported ${ }^{5}$ that among 23 patients treated with daily RMP together with PTH in a daily dose of at least $500 \mathrm{mg}, 4$ developed jaundice, and 3 manifested abnormalities of liver function without clinically evident jaundice.

Among leprosy patients treated at the Institut Marchoux, Bamako, Mali, with a regimen consisting of dapsone $100 \mathrm{mg}$, RMP $600 \mathrm{mg}$ and PTH $500 \mathrm{mg}$, each administered daily, ${ }^{6} 4$ of 12 patients became jaundiced and 1 of the 4 died. ${ }^{7}$ One study ${ }^{8}$ has found 7 cases of hepatitis, 5 of them clinically jaundiced, among 54 patients treated with daily RMP together with $500 \mathrm{mg}$ daily of either PTH or ethionamide. After the dosage of the thioamide had been reduced to $5 \mathrm{mg} / \mathrm{kg}$ body weight, no additional cases of jaundice occurred, although elevated serum transaminase levels were noted in a few patients.

The dosages of RMP and R-76-1 were rather low in the present study, as these drugs were usually administered by a monthly schedule. No toxic effects of monthly RMP have been reported..$^{9}$ A number of patients with multibacillary leprosy have been treated with R-76-1 as monotherapy in a daily dosage of 150 $\mathrm{mg}$ for 6-18 months, or in a monthly dosage of $1200 \mathrm{mg}$, with negligible hepatotoxicity. ${ }^{3}$ Because there has been no controlled clinical trial with combined regimens of prothionamide and either RMP or R-76-1, we cannot confirm or deny the possibility that R-76-1 may predispose to more serious hepatotoxicity than does RMP when combined with daily prothionamide. However, although the total dosage of R-76-1 was highest in Hai-an Group B patients, there was no 
difference of the frequency of liver injury among the 3 groups of Hai-an patients. Therefore, the liver injury reported in this present study cannot be attributed to the hepatotoxicity of RMP or R-76-1.

Thus, it appears that, among the drugs administered in the present study, the drug responsible for the liver injury is PTH. However, it is uncertain whether the hepatotoxicity was simply a toxic effect of $\mathrm{PTH}$, or whether it represented a summation of the effect of PTH combined with that of RMP or R-76-1, even though the latter 2 drugs were administered only in low dosage.

We cannot exclude the possibility that, despite normal liver function tests at the onset of treatment, some pre-existing liver damage was present among the patients of this study. All of the patients were active (i.e. skin-smear-positive) multibacillary cases. From the literature, leprous granulomata as well as acid-fast bacilli have frequently been demonstrated in the livers of leprosy patients. ${ }^{11,12}$ The leprous granulomata were found in $21 \%$ of patients with tuberculoid leprosy and in $62 \%$ of patients with lepromatous leprosy; further, granulomata were also discovered in the livers of 22 of 101 leprosy patients who had no acid-fast bacilli in skin smears. ${ }^{11}$ The liver lesions in some patients were characterized by early cirrhosis. ${ }^{12}$ In addition, a rise of transaminase activity, although not as serious as that in our patients, has also been reported in patients under dapsone treatment. ${ }^{13}$ Therefore, pre-existing liver damage may have been an important predisposing factor to hepatotoxicity with prothionamide. Liver damage is rare among patients with tuberculosis, except in cases of miliary tuberculosis. ${ }^{14}$ This could be one of the explanations of why the hepatotoxicity of prothionamide appears to be less serious in tuberculosis patients than in leprosy patients.

To be safe, we have temporarily suspended treatment by all of the regimens employed in this study. On the other hand, PTH exerts powerful bactericidal activity against $M$. leprae (10; Ji Baohong et al., unpublished data). Considering that a significant proportion of Chinese patients will not accept CLO, it does not appear justified to abandon PTH as a component of combined drug regimens. In fact, PTH may still be used with safety, provided the patients are carefully supervised and followed up. We are planning additional controlled trials, to evaluate further the hepatotoxicity of PTH alone and in combination with RMP. In order to detect toxicity as early as possible, tests of liver function will be performed monthly during the first 6 months of the combined treatment.

\section{References}

${ }^{1}$ Fifth Report of the WHO Expert Committee on Leprosy. WHO Technical Report Series No. 607, 1977.

${ }^{2}$ Report of a WHO Study Group. Chemotherapy of leprosy for control programmes. WHO Technical Report Series No. 675, 1982.

3 Ji Baohong, Chen Jiakun, Zhou Daohai. Chemotherapeutic studies of leprosy. VI. The therapeutic effect of R-76-1 in multibacillary leprosy. Chinese J Dermatol, 1983; 16: 19-23. 
4 Ji Baohong, Chen Jiakun, Zhang Jialin, Hou Yuhong, Ni Guoxing, Zhang Renbao. Secondary dapsone resistant leprosy in Shanghai Municipality. Lepr Rev, 1983; 54: 197-202.

${ }^{5}$ Lesobre R, Ruffine J, Teyssier L, Achard F, Brefort G. Les icterus en cours du traitement par la rifampicine. Rev Tuberc Pneumol, 1969; 33: 393-403.

${ }^{6}$ Report of the third meeting of the Scientific Working Group on Chemotherapy of Leprosy (THELEP) of the UNDP/World Bank/WHO Special Programme for Research and Training in Tropical Diseases. Int J Lepr, 1981; 49: 431-6.

7 WHO publication TDR/AR(5)/81.8-LEP. Annual report of the Special Programme for Research and Training in Tropical Diseases. Chapter 8, Leprosy.

8 Cartel JL, Millan J, Guelpa-Lauras CC, Grosset JH. Hepatitis in leprosy patients treated by a daily combination of dapsone, rifampin and a thioamide. Int J Lepr, 1983; 51: 461-65.

9 Rees RJW. Rifampicin: the investigation of a bactericidal antileprosy drug. Lepr Rev, 1975; 46 Suppl: 121-4.

10 Ji Baohong, Tong Quangui, Wang Shiyu, Ni Guoxing. Therapeutic studies on experimental leprosy by mouse footpad model. Chinese J Dermatol, 1980; 13: 24-8.

${ }^{11}$ Karat ABA, Job CK, Rao PSS. Liver in leprosy: histological and biochemical findings. Brit Med $J, 1971$; i: $307-10$.

12 Agarwal SC, Maheshwari HB, Mittal MM, Kumar S. A histological study of liver lesions in leprosy. Indian J Med Res, 1973; 61: 389-95.

${ }^{13}$ Robins K, Vijayakumar T, Gopinath T, Vasudevan DM. Liver in leprosy-I Functional changes. Lepr India, 1980; 52: 416-22.

14 Rich AR. The Pathogenesis of Leprosy, 2nd printing, pp. 311-12. Springfield, Ill.: Charles C. Thomas, 1946. 